\title{
Bildungsdisparitäten und Ethnizität:
}

\author{
Zur schulischen Situation \\ der Gitano-Minderheit in Spanien
}

\begin{abstract}
Summary: Spanish Gitanos or Cale form one of the largest Romany minorities in the EU. While they lived at the margins of Spanish society over centuries, their living and educational conditions have improved considerably within the last three decades. This article focuses on the educational situation of the Gitanos by incorporating empirical studies published on that topic in the recent years in Spain. It can be shown that the schooling of this ethnic minority has improved on the one hand, while considerable educational disparities continue to exist in comparison to the majority population on the other hand. In conclusion, some recent challenges are discussed which are of relevance for this specific case of educational inequality.
\end{abstract}

Резюме: Испанские цыгане или кале образуют одну из крупнейших цыганских меньшинств ЕС. Если столетиями они жили на краю испанского общества, то за последние три десятилетия условия их жизни и образования значительно улучшились. Статья фокусирует свое внимание на образовательной ситуации цыган и анализирует эмпирические исследования, которые были в последние годы опубликованы в Испании по данной тематике. В статье показано, что, с одной стороны, образовательная ситуация данного этнического меньшинства улучшилась, но, с другой стороны, как и прежде, существуют серьезные образовательные диспропорции в отношении населения меньшинства. В заключении рассматриваются некоторые теоретические задачи, которые для данного специального случая этнического равноправия в области образования являются релевантными.

Zusammenfassung: Spanische Gitanos oder Calé bilden eine der größten Roma-Minderheiten in der EU. Während sie über Jahrhunderte am Rande der spanischen Gesellschaft lebten, haben sich ihre Lebens- und Bildungsbedingungen in den letzten drei Dekaden stark verbessert. Der Artikel fokussiert auf die Bildungssituation der Gitanos und arbeitet empirische Studien auf, die in den letzten Jahren in Spanien zum Thema veröffentlicht wurden. Es kann gezeigt werden, dass sich die Bildungssituation dieser ethnischen Minderheit einerseits verbessert hat, andererseits aber nach wie vor gravierende Bildungsdisparitäten zur Mehrheitsbevölkerung bestehen. Abschließend werden einige aktuelle Herausforderungen diskutiert, die für diesen speziellen Fall ethnischer Bildungsungleichheit relevant sind.

\section{Einführung}

Europaweit dürfte es kein prägnanteres Beispiel ethnischer Bildungsdisparitäten geben, als die Bildungsnachteile jener Minderheiten, die von Seiten der EU-Administration mittlerweile unisono als „Roma“ bezeichnet werden, und für die in verschiedenen Ländern, Sprachen sowie gruppenbezogen weitere Ethnonyme wie Gitanos, Gypsies, Sinti, Zigeuner, Manouches, Lovara, Kalderasch u. a. geläufig sind. Vielerorts gehen diese Bildungsnachteile mit ausgesprochen prekären Lebensbedingungen und konfliktträchtigen interethnischen Beziehungen einher. Wo Studien und Statistiken vorliegen, dokumentieren sie durchgehend, dass Roma zu den gesellschaftlich am stärksten marginalisierten Bevölkerungssegmenten zählen. Zweifelsohne existieren zwischen den Lebensbedingungen von Roma verschiedener Staaten große Unterschiede, in denen sich nicht zuletzt EU-weite Unterschiede im Lebens- und Menschenrechtsstandard widerspiegeln. Ebenso wenig dürfen die Unterschiede innerhalb dieser Gemeinschaften übersehen werden: Einige Roma sind als Politiker in 
europäische Parlamente gewählt worden, als Künstler und Sportler in den Medien präsent oder als selbstständige Unternehmer erfolgreich. Dennoch belegen alle verfügbaren Erhebungen, dass sie als demographische, paneuropäische Kategorie in allen wichtigen Lebenschancen-Indikatoren wie der Lebenserwartung, dem Gesundheitszustand, der Alphabetisierung, den Bildungsabschlüssen und den beruflichen Qualifikationen, dem Einkommen oder den Wohnbedingungen, deutlich schlechter gestellt sind als die Angehörigen der jeweiligen gesellschaftlichen Mehrheit. „Ein Großteil der Roma", so fasste der EU-Kommissionspräsident José Manuel Barroso dieses Phänomen anlässlich des ersten europäischen Gipfeltreffens zur Lage der Roma im September 2008 zusammen, „lebt unter Bedingungen, die im Europa des 21. Jahrhunderts einfach nicht akzeptabel sind" (zitiert nach Pape, 2008, 1).

Dem Bildungsgeschehen wird für den Integrationsprozess der Roma ein herausragender Stellenwert zugesprochen: Zum einen, weil die Lebenschancen und die Lebensführung von Individuen wie auch von sozialen Gruppen in vielerlei Hinsicht von ihrer Bildung profitieren; zum anderen, weil moderne, in den globalen Wettbewerb eingebundene Gesellschaften auf kompetente, kreative und Wissen schaffende Bürger angewiesen sind. Es überrascht daher nicht, wenn Politik, Schulpraxis und Forschung gleichermaßen das Bildungsgeschehen als Schlüsselfaktor für die RomaIntegration auf lokaler, nationaler wie europäischer Ebene identifiziert haben.

Vorliegender Artikel thematisiert die Problematik ethnischer Bildungsdisparitäten am Fallbeispiel spanischer Gitanos bzw. Calé. Als Nachkommen von seit dem 15. Jahrhundert in Spanien eingewanderten, ehemals Romani sprechenden Gruppen gelten die Gitanos nicht nur als Spaniens größte ethnische Minderheit, sondern zudem (unter Vorbehalt statistischer Unsicherheiten) als größte Gruppe der Roma-Kategorie in Westeuropa. Auch EU-weit leben nur in Rumänien und Bulgarien mehr Roma (obgleich der prozentuale Anteil in mehreren osteuropäischen Staaten höher als in Spanien liegt). ${ }^{1}$ Gitanos sind heutzutage spanischsprachig und sedentär. Damit werden aus vergleichender Betrachtungsweise zwei Faktoren (Mehrsprachigkeit und Nichtsesshaftigkeit), die oftmals als Gründe für die Bildungsnachteile bestimmter ethnischer Minoritäten im Allgemeinen, der Roma im Besonderen angeführt werden, ausgeschlossen. Von besonderer Relevanz ist das Beispiel jedoch in empirischer Hinsicht: Ihre Lebens- und Bildungssituation ist wissenschaftlich ungleich besser erforscht als die von Roma in anderen EU-Staaten. Sieht man von den frühen folkloristischen und sprachwissenschaftlichen Publikationen des 19. Jahrhunderts ab, setzt die empirische Forschung zur Lebens- und Schulsituation der Gitanos durch Ethnologen, Soziologen und Erziehungswissenschaftler in den 1970er Jahren ein. Gegenwärtig stellen die Gitanos die am besten empirisch erforschte ethnokulturelle Minderheit Spaniens dar (vgl. z. B. Abajo Alcalde \& Carrasco, 2004; Alfageme Chao \& Martínez Sancho, 2004; Asensio Belenguer, 2011; Asociación Secretariado General Gitano, 2001; Caselles Pérez, 2008, 2013; Gay y Blasco, 1999, 2005; Equipo EINA, 2003; Fernández Enguite, 1999; Fundación Secretariado Gitano, 2006; Gamella, 1996, 1999; 2012; García Pastor, 2009; Garreta Bochaca, 2003; Giménez Adelantado, 2003; IFIIE \& Instituto de la Mujer, 2010; Instituto de la Mujer, CIDE \& Fundación Secretariado Gitano, 2006; Lalueza, Crespo, Pallí \& Luque, 2001; Laparra (Hrsg.), 2007, 2011; López de la Nieta, 2011; Martínez Sancho, Giménez Adelantado \& Alfageme Chao, 2001; San Román, 1990; Vázquez et al., 1990 [1978]).

1 Die verlässliche statistische Zählung von „Roma“ in Europa ist aus verschiedenen Gründen, die hier nicht ausgeführt werden, äußerst problematisch. Nimmt man die Durchschnittswerte der jeweils niedrigsten und höchsten Zählungen zu Hand, leben in Bulgarien, Mazedonien, Rumänien, Serbien, der Slowakei und Ungarn mit zwischen 7 und über 10 Prozent anteilig am meisten Roma. In absoluten Zahlen leben die meisten in der Türkei, der Russischen Föderation, Rumänien, Bulgarien und Spanien (siehe die fortlaufend aktualisierte Statistik unter URL http://www.coe.int/t/dg3/ romatravellers/default_en.asp (letztes Update vom 14.9.2010). 


\section{Gitanos in Spanien}

Etymologisch wird die Bezeichnung Gitanos von dem Wort egipcianos („Ägypter“) abgeleitet. In dieser Form findet es sich in den ältesten Schriftdokumenten, die von der Ankunft dieser Fremden auf der Iberischen Halbinsel berichten und von denen man annahm, sie seien Pilger aus „Klein-Ägypten“. Ende des 15. und Anfang des 16. Jahrhunderts existierten noch weiter Bezeichnungen - ein katalanisches Gesetz von 1512 etwa spricht von „persones ques dirian vulgarment boemians y sots noms de boemians grechs o egipcians“ („Personen, welche man gemeinhin Böhmer nennt und die Namen tragen wie Böhmer, Griechen oder Ägypter") ${ }^{2}$ - der Begriff Gitanos setzte sich indes durch und wurde in Spanien zum Sammelbegriff für Angehörige dieser Minderheit. Parallel wird fernerhin das Ethnonym Calé (in der deutsch- und englischsprachigen Literatur zumeist Kalé geschrieben) verwendet, das von dem Romani-Wort für "schwarz“ abgeleitet wird. Nach Matras (2003) war Calé/Kalé eine vormals weitverbreitete Selbstbezeichnung und fand sich neben Spanien auch in Großbritannien, Deutschland, Böhmen und Skandinavien. ${ }^{3}$

Nachdem die regionalen Herrscher den durchreisenden Fremden in den ersten Jahrzehnten mit großem Wohlwollen begegnet waren, setzte mit der königlichen Pragmática von 1499 eine Kette von gesetzlichen Maßnahmen ein, die entweder auf die Ansiedlung und Assimilation oder die Ausweisung der Gitanos abzielten. Bereits der Umstand, dass derartige Verordnungen zu Dutzenden neu erlassen wurden, zeigt an, dass ihre Durchsetzung wenig erfolgreich war. Erst die Pragmática von Karl III. aus dem Jahr 1783 schloss die Reihe von Gesetzen und erklärte die Gitanos zu spanischen Bürgern mit gleichen Rechten und Pflichten.

Geschichtliche Erfahrungen haben Spuren im kollektiven Selbstbild vis-à-vis der Mehrheitsgesellschaft hinterlassen. Dennoch wäre es unangemessen, die gitanische Gegenwartskultur ausschließlich auf historische Reaktionen auf die Mehrheitsgesellschaft zu reduzieren. Heutzutage teilen Gitanos in kultureller Hinsicht viele Charakteristika mit der spanischen Mehrheitsbevölkerung. Zugleich haben sie sich ein ausgeprägtes Wir-Bewusstsein gewahrt, dass von speziellen sozialen Strukturen (Verwandtschaftsbeziehungen, Geschlechterverhältnisse, Ehe und Elternschaft) und kulturellen Praxisformen und Idealen (Endogamie, Ehre, Virginität, Virilität) genährt wird. Die Wirtschaftsweisen der Gruppen waren ursprünglich an ihre Mobilität angepasst, wobei Erfahrungen in einem Handwerk oder Gewerbe innerhalb der Familie vom Vater an die Söhne (z.T. wohl auch von der Mutter an die Töchter) weitergegeben und durch weitere flexible Einkommensquellen ergänzt wurden. Die gruppenspezifischen Wirtschaftsweisen wiesen Ähnlichkeiten mit dem indischen Kastensystem auf, vor allem mit der ökonomischen Spezialisierung der jatis, bzw. sind ursprünglich aus dem Kastensystem hervorgegangen. ${ }^{4}$

In den letzten drei Jahrzehnten setzten weitreichende Transformationen der Gitano-Kultur ein. Die Behauptung gitanischer Identität, kulturelle Hybridisierung und individuelles Assimilationsbestreben stehen heutzutage nebeneinander. Ebenso ist die gesellschaftliche Lage der Gitanos durch eine zunehmende Heterogenität gekennzeichnet. Konnte man vor einer oder zwei Generationen noch von einer weitgehenden Marginalisierung und Unterschichtung sprechen, sind gegenwärtig

Corts de Montsó (1512): "De la expulcio dels boemians“ (vgl. URL www.mcu.es/archivos/lhe/).

Angehörige der spanischen Mehrheitsbevölkerung werden in diesem Kontext umgangssprachlich, z.T. aber auch in der Forschung, als payos bezeichnet, ein durchaus despektierlicher Begriff, der die auch anderenorts übliche dichotome wir/sie-Distinktion zwischen ursprünglich durchreisenden Fremden und einer sesshaften Landbevölkerung widerzuspiegeln scheint.

4 Wie linguistische, ethnologische und nunmehr auch humangenetische Studien zeigen, entstammen die europäischen Roma ursprünglich dem indischen Subkontinent, vor allem dem nordwestlichen Indien, und sind vermutlich Nachkommen von Kastengruppen, die ihre Heimat vor etwa 1000-1200 Jahren verlassen hatten. 
mehr Gitanos in die untere Mittelschicht aufgestiegen. „Die spanischen Gitanos“, schreibt Lucía Petísco (2012) von der Fundación Secretariado Gitano, „haben sich in drei Jahrzehnten mehr gewandelt als in sechs Jahrhunderten zuvor".

\section{Demographische Angaben}

Wie viele Spanier sich heutzutage der Gitano-Minderheit zurechnen, kann lediglich näherungsweise beziffert werden. Schätzungen und Hochrechnungen reichen von 500000 bis 1 Million Personen, was bis zu 2,1 Prozent der Gesamtbevölkerung entspräche. Die meisten Autoren gehen von einem Mittelwert zwischen diesen beiden Polen aus. Die spanische Regierung spricht aktuell von 725000 bis 750000 Personen bzw. etwa 1,5 Prozent der Bevölkerung (Ministerio de Sanidad, Servicios Sociales e Igualdad, 2012, 11). Der detaillierte Vergleich verschiedener Statistiken zur GitanoBevölkerung zeigt starke Abweichungen, wobei die Zahlen für einige der Autonomen Gemeinschaften Differenzen von bis zu 67 Prozent aufweisen (Laparra \& del Pozo, 2007, 14). Sicher ist, dass die regionale Verteilung der Gitanos stark variiert. Mit bis zu 52 Prozent leben die meisten im südspanischen Andalusien, wo sie in zahlreichen Dörfern im Umland von Granada - etwa in den Gemeinden Iznallos, Lanjarón, Colomera oder Deifontes - bis zu einem Drittel der Ortsbewohner ausmachen. Weitere demographische Kristallisationspunkte bilden großstädtische Zentren wie Madrid, Barcelona, Sevilla, Valencia und Saragossa. Mancherorts gehen diese Schwerpunkte auf die erzwungene Niederlassung zurück, die ab dem 17. Jahrhundert forciert wurde; anderenorts resultieren sie aus der Landflucht im Kontext der beschleunigten Urbanisierung und Industrialisierung der 1950er Jahre. Die geringste Anzahl wird für die Kanaren, die nordafrikanischen Enklaven Ceuta und Melilla sowie Asturien und Kantabrien angeführt.

Tabelle 1: Schätzung der Größenordnung und der regionalen Verteilung der Gitano-Bevölkerung 1999

\begin{tabular}{|l|c|c|c|}
\hline \multicolumn{1}{|c|}{ Region } & Gesamtbevölkerung & Gitanos absolut & Gitanos in \% \\
\hline Andalusien & 7236459 & 286110 & 3,95 \\
\hline Aragon & 1183234 & 18209 & 1,54 \\
\hline Asturien & 1081834 & 4780 & 0,44 \\
\hline Balearen & 796483 & 5423 & 0,68 \\
\hline Baskenland & 2098628 & 11675 & 0,56 \\
\hline Ceuta und Melilla & 132225 & 2030 & 1,54 \\
\hline Extremadura & 1069419 & 11318 & 1,06 \\
\hline Galizien & 2724544 & 13741 & 0,50 \\
\hline Kanaren & 1630015 & 854 & 0,05 \\
\hline Kantabrien & 527137 & 4021 & 0,76 \\
\hline Kastilien-La Mancha & 1716152 & 33552 & 1,96 \\
\hline Kastilien und León & 2484603 & 28339 & 1,14 \\
\hline Katalonien & 6147610 & 52937 & 0,86 \\
\hline La Rioja & 263644 & 7361 & 2,79 \\
\hline Madrid & 5091336 & 59082 & 1,16 \\
\hline Murcia & 1115068 & 33006 & 2,96 \\
\hline Navarra & 530819 & 5954 & 1,12 \\
\hline Valencia & 4023441 & 52455 & 1,30 \\
\hline Gesamt & 39852651 & 630847 & 1,58 \\
\hline
\end{tabular}


Im Vergleich zur Mehrheitsbevölkerung weisen die Gitanos ein höheres Bevölkerungswachstum auf. Berechnungen der Bevölkerungsentwicklung kommen zu dem Schluss, dass die Zahl der Gitanos seit dem späten 16. Jahrhundert trotz Repressionen um das 30 -fache gestiegen ist, während die spanische Bevölkerung von vier auf 40 Millionen wuchs (Ringold, Orenstein \& Wilkens, 2005, 164). Für Gitanos in Andalusien ging man in den 1990er Jahren von einer Geburtenrate von 23,8 Geburten auf 1000 Personen aus, verglichen mit 13 Geburten auf 1000 unter den andalusischen NichtGitanos und zehn Geburten für die spanische Gesamtbevölkerung (Gamella, 1996, 75-86). Ein weiteres demographisches Charakteristikum ist die relativ junge Altersstruktur. 48,8 Prozent der Gitanos sind jünger als 24 Jahre, bei der spanischen Gesamtbevölkerung sind dies lediglich 25,2 Prozent. Zudem gibt es einen deutlich geringeren Anteil von Gitanos, die älter als 65 Jahre sind (3,8 Prozent gegenüber 17,1 Prozent) (Fundación Secretariado Gitano, 2012, 21).

\section{Integrationspolitik für Gitanos}

Seit Ende der 1970er Jahre wurden mit der Demokratisierung und Modernisierung des spanischen Staates erste Schritte zur Bekämpfung der Armut und der gesellschaftlichen Randstellung der Gitano-Minderheit eingeleitet. Wegweisend war das 1984 beschlossene und 1989 implementierte Nationale Entwicklungsprogramm für das Volk der Gitanos (Programa de Desarrollo Gitano). Für verschiedene Autonome Gemeinschaften liegen regionale Integrationspläne vor, welche die vielfältigen lokalen Integrationsinitiativen koordinieren und mit dem nationalen Entwicklungsplan abstimmen. Der Europäischen Sozialfonds beteiligt sich maßgeblich an Regierungsprogrammen und Initiativen von Nichtregierungsorganisationen, die sich für die Anhebung des Bildungsstandes, Ausbildungsplätze, Wohnungsbauprojekte, eine Verbesserung der medizinischen Versorgung, Antidiskriminierungskampagnen und interkulturelle Projekte einsetzen. Aus dem Etat des nationalen Aktionsplans zur sozialen Inklusion (Plan Nacional de Acción para la Inclusión Social) werden ebenfalls Fördermittel für sozioökonomisch schlecht gestellten Gitano-Familien bereitgestellt. 2005 wurde per Königlichem Dekret ein Staatlicher Rat des Gitano-Volkes (Consejo Estatal del Pueblo Gitano) als beratendes Gremium aus Gitano-Verbänden, NGOs und staatlichen Akteuren ins Leben gerufen. ${ }^{5}$ Ähnliche Gremien gibt es mittlerweile auch auf regionaler Ebene (Andalusien, Baskenland, Extremadura, Katalonien). 2007 folgte die Einrichtung eines staatlichen Instituts für die Kultur der Gitanos (Instituto de Cultura Gitana), das dem Kulturministerium zugeordnet ist und unter dem Motto España también es gitana ("Spanien ist auch gitanisch“) die Erforschung und Entwicklung der Gitano-Kultur fördert. ${ }^{6} 2012$ wurde eine Nationaler Strategieplan zur sozialen Inklusion der GitanoBevölkerung für die Jahre 2012 bis 2020 vorgestellt, der indikatorengestützt die Verbesserung der Lebensbedingungen der Gitanos in den Bereichen Bildung, Arbeitsmarkt, Wohnsituation und Gesundheit voranbringen soll (Ministerio de Sanidad, Servicios Sociales e Igualdad, 2012). Zudem gibt es ein breites Spektrum zivilgesellschaftlicher Organisationen, die (oft in Zusammenarbeit mit staatlichen Stellen oder vom Staat mitfinanziert) für die Gitanos arbeiten. Die größte und einflussreichste Organisation ist die Fundación Secretariado Gitano (FSG), die als gemeinnützige Stiftung für die Entwicklung der Gitano-Minderheit in Gesamtspanien aktiv ist. Die FSG wurde 1982 als Asociación Secretariado General Gitano gegründet, Vorläuferaktivitäten reichen aber bis in die 1960er Jahre zurück. Seit 2001 ist die FSG als Stiftung mit Hauptsitz in Madrid eingetragen. Sie verfügt heute über 77 Ortsbüros in 14 Autonomen Gemeinschaften. Die Stiftung verwaltet ein Jahresbudget von rund 20 Millionen Euro, was sie zu der wichtigsten nichtstaatlichen Organisation der Gitano-Entwicklungsarbeit macht. 2005 unterzeichneten das spanische Bildungsministerium und die FSG ein gemeinsames Strategiepapier zur Verbesserung des Zugangs und der Integration der

5 Nähere Informationen zum Rat des Gitano-Volkes finden sich auf der Webseite des Ministeriums für Gesundheit, soziale Dienste und Gleichheit unter http://www.msssi.gob.es/.

6 Siehe http://www.institutoculturagitana.es/ 
Gitano-Schüler im Bildungswesen. Aktuell kooperiert die Stiftung mit Roma-Organisationen in Osteuropa, wo sie angesichts ihrer professionellen Arbeit als Ideengeber und Vorreiter geschätzt wird. Nach Salinas $(2007,34)$ gibt es daneben rund weitere 600 Lobbygruppen, die sich für die Gitanos einsetzen, darunter 40 für Gitano-Frauen. ${ }^{7}$ Zwei ethnopolitische Gitano-Parteien, die Partido Nacionalista Caló und die Alianza Romaní, blieben bedeutungslos.

\section{Wohnsituation}

Seit den 1960er Jahren hat sich die Wohnsituation für Gitanos schrittweise verbessert. Noch unter Franco begann die staatliche Wohnungsbaupolitik, ungenehmigte Barackensiedlungen (chabolas) abzureißen, Höhlenwohnungen (cuevas) zu räumen und Gitanos (wie auch andere Spanier, die unter ähnlichen Bedingungen lebten) in neu errichteten Siedlungen des sozialen Wohnungsbaues unterzubringen. Barackensiedlungen und Elendsviertel gehören heutzutage weitgehend der Vergangenheit an. Nur noch 4,4 Prozent der Unterkünfte stellten 2007 entsprechende Baracken, Höhlen oder Wohncontainer dar (Fundación Secretariado Gitano, 2008/2009). Allerdings haben die in den späten 1960er, 1970er und 1980er Jahren errichteten Siedlungen des sozialen Wohnungsbaues mancherorts innerstädtische Segregationsprozesse provoziert. Die residenzielle Konzentration von Gitano-Familien trug in einigen Gegenden dazu bei, dass es verstärkt zu Blutfehden zwischen konkurrierenden Familienverbänden (razas) kam und sich später mafiaähnliche Netzwerke entwickelten (Gay y Blasco, 2005, 165). Einige Wohnviertel verwahrlosten, was sich in einer Zunahme des Drogenhandels und -konsums, der Gewalt oder der Vermüllung von Straßenzügen zeigte. Quartiere wie das zwischen 1968 und 1977 errichte Polígono Sur („Las Tres Mil Viviendas“) in Sevilla, die Anfang der 1970er Jahre errichte Trabantenstadt La Mina in der Metropolregion Barcelonas oder die Barackensiedlung Son Banya am Rande von Palma de Mallorca erlangten einen notorischen Ruf. Obgleich diese Siedlungen ursprünglich mit öffentlichen Mitteln finanziert wurden, um den chabolismo („Verslumung") zu bekämpfen, spricht man seit den 1990er Jahren auch von einem chabolismo vertical („vertikale Verslumung“).

\section{Arbeitsmarktsituation}

Nach wie vor unterscheidet sich die Arbeitsmarktsituation der Gitano-Bevölkerung deutlich von der Beschäftigungssituation der Mehrheitsbevölkerung. Es kommt seltener vor, dass Gitanos über regelmäßige Erwerbseinkünfte verfügen oder sich in einem Angestelltenverhältnis befinden. In manchen Regionen praktiziert die Mehrheit noch heute ,traditionelle' Erwerbsformen, wie den ambulanten Handel, das Einsammeln von Wertstoffen oder die Saisonarbeit in der Landwirtschaft. Diese Erwerbstätigkeiten sehen sich jedoch zunehmend mit bürokratischen Beschränkungen und Konkurrenz konfrontiert. Abfallverordnungen, moderne Recyclingfirmen, Steuern, Zuwanderung von Erntehelfern aus dem Ausland oder chinesische und indische Markthändler, die ihre Waren über effizientere Netzwerke beziehen, dezimieren die Einkommensoptionen erheblich. Wirtschaftlich besser gestellt sind Gitanos, die im Antiquitätenhandel, als Ladenbesitzer oder als Musiker tätig sind. Bei genannten Erwerbsbereichen handelt es sich häufig um Berufsfelder mit familialen bzw. verwandtschaftlichen Traditionen, in welche die Familienmitglieder im Laufe ihres Heranwachsens sozialisiert werden, um später selbst etwas zum Familienunternehmen beizutragen. In der einst-

\footnotetext{
7 Die Mehrzahl dieser gitanischen Organisationen sind auf familialer Basis organisiert und verfügen über wenige Mitglieder. Zu den überregionalen Organisationen neben der FSG zählen die Asociación Nacional Presencia Gitana, Unión Romaní, Asociación de Enseñantes con Gitanos, Asociación Socio-cultural de las Minorías Étnicas „Unga“, Federación de Asociaciones Gitanas para la integración laboral y social, promoción y desarrollo del pueblo gitano „Cali“ und die Federación Nacional de Asociaciones de Mujeres Gitanas „Kamira“.
} 
mals boomenden Baubranche arbeiteten seit den 1990er viele Gitanos, die mit der Immobilien- und Wirtschaftskrise ab 2007 ihre Arbeit verloren. Karitative Verbände und Lobby-Organisationen bilden ebenfalls neuere Berufsfelder. Berufe, die einen individualisierten und institutionalisierten Ausbildungsgang voraussetzen, gewinnen erst langsam an Bedeutung. Hierzu tragen v.a. verschiedene Beschäftigungsprogramme für Gitanos bei, wie das seit 2000 von der FSG verantwortete ACCEDER-Programm, welches die Arbeitsmarktintegration von Gitanos zum Ziel hat, indem Fortbildungen, firmeninterne Schulungen, Praktika und Jobs angeboten und vermittelt werden. Seit 2011 sind 24 regionale ACCEDER-Büros als Personalvermittlungsagenturen anerkannt. ${ }^{8}$ Stark gefährdet werden diese ersten positiven Entwicklungen auf dem Beschäftigungssektor durch die Wirtschaftskrise der letzten Jahre, die die Lage vieler Gitano-Familien verschlechtert hat. (Caselles Pérez, 2013; Fundación Secretariado Gitano, 2013).

\section{Soziale Probleme}

$\mathrm{Zu}$ den sozialen Problemen, die in einigen Gitano-Gemeinschaften alarmierend sind, zählen der Konsum von Drogen und die Kriminalität. 1999 stellten Gitano-Frauen ein Viertel der Insassinnen der spanischen Strafanstalten und waren damit deutlich überrepräsentiert. Die durchschnittliche Haftstrafe der inhaftierten Gitanas betrug 6,7 Jahre. 59,8 Prozent von ihnen waren (funktional oder gänzlich) analphabetisch, 45 Prozent hatten illegale Drogen konsumiert und 87,3 Prozent der inhaftierten Gitanas waren Mütter von durchschnittlich drei Kindern (Martín Palomo, 2002). Vergleichbare Informationen zu Gitano-Männern fehlen. Erklärungsversuche mit Begriffen wie „Kriminalisierung" und „Diskriminierung“ sind heutzutage schnell zur Hand, suggerieren allerdings, dass moderne Gesellschaften ein Interesse an der Alimentierung wirtschaftlich unproduktiver, inhaftierter und Drogen konsumierender Mütter hätten.

\section{Bildungspolitische Maßnahmen für Gitanos seit den 1970er Jahren}

Bis weit in die 1970 er Jahren besuchte nur eine Minderheit der Gitano-Kinder regelmäßig die Schule und noch weniger erlangten einen Abschluss. 1978 hatte lediglich die Hälfte der GitanoBevölkerung im Alter über 6 Jahren zeitweise eine Schule besucht (Asociación Secretariado General Gitano, 1979/1990, 144f.). Die erste postfranquistische, bildungspolitische Initiative für GitanoKinder bestand in der Gründung sogenannter Brückenschulen (span.: escuelas puentes), die nach einem Abkommen zwischen der katholischen Kirche und dem Bildungsministerium 1978 ins Leben gerufen wurden. Man konnte sich dabei auf das noch unter Franco am 4. August 1970 erlassene Ley General de Educación y Financiamiento de la Reforma Educativa (kurz: LGE) berufen, welches eine quantitative wie qualitative Ausweitung und grundlegende Modernisierung des spanischen Bildungssystems angestrebte. Mit dem Gesetz wurde u.a. die Schulpflicht vom 6. bis zum 14. Lebensjahr eingeführt. Art. 51 des LGE legte die Einrichtung spezieller Übergangsschulzentren für Kinder mit „Defiziten“ und „Anpassungsproblemen“ nahe. Oft handelte es sich bei den Brückenschulen um separate Klassenräume in Schulen in ärmeren Landesteilen und in städtischen Quartieren, in denen

8 Von 2000-2006 standen ACCEDER über 64 Millionen Euro zur Verfügung, die zu etwa Zweidrittel aus dem Europäischen Sozialfonds (ESF) und zu einem Drittel vom spanischen Arbeitsministerium, den Autonomen Gemeinschaften, den Kommunen und privaten Spenden aufgebracht wurden. In dem Zeitraum nahmen 35304 an dem Programm teil, darunter zu 70 Prozent Gitanos und etwas über die Hälfte Frauen. Angesicht des Erfolges des Programmes wurde es mehrfach vom Europäischen Sozialfonds, der Europäischen Kommission und den Vereinten Nationen ausgezeichnet. Für die Jahre 2007-2013 wird das Programm mit fast 42 Millionen Euro subventioniert. Der englischsprachige Gesamtreport für die Jahre 2000-2006 findet sich unter URL http://www.gitanos.org/publicaciones/memoriaacceder2007/english/. 
viele Gitano-Familien lebten. Sie zielten darauf ab, die Kinder auf den Besuch der Regelschulen vorzubereiten und waren folglich als pädagogische Übergangsmaßnahme gedacht. Zudem richteten sich diese Schulangebote nicht an alle Gitano-Kinder, sondern nur an jene, für die besonderer Nachhol- und Förderbedarf diagnostiziert wurde (Fresno, 2001/2002, 73). Zu den Vorteilen des puente-Konzeptes zählte man den Umstand, dass die Schulen in das örtliche Lebensumfeld der Gitano-Familien eingebettet waren, eine sozial und kulturell weitgehend homogene Schülerschaft bedienten und von Lehrpersonal geleitet wurden, das mit dem Programm sympathisierte. Bis zum Auslaufen dieser Maßnahmen im Jahr 1986 zählte man 182 Brückenschulen, in denen pro Schuljahr insgesamt nie über 6000 Schüler eingeschrieben waren (Salinas, 2009, 175).

Mehrere Gründe trugen zur Beendigung dieses schulischen Modells bei: Zum einen hatte sich die Bildungssituation der Gitanos nicht ausreichend verbessert und die Segregation von GitanoKindern in eigenen Klassenräumen oder Gebäuden war der angestrebten Integration in die Regelschulen abträglich. Vor allem aber ließ die bildungspolitische Neuausrichtungen des Bildungsministeriums, die sich in dem Königlichen Dekret 1174/83 zur „kompensatorischen Erziehung“ von 1983 und dem Organrecht („Verfassungsrecht") zum Recht auf Bildung (Ley Orgánica del Derecho a la Educación) von 1985 niederschlug, das Konzept der Brückenschulen veraltet erscheinen. Erklärtes Ziel war es fortan, Bildungschancengleichheit für alle benachteiligten Schüler und Regionen zu erreichen. Dazu wurden die infrastrukturellen und pädagogischen Bildungsbedingungen in ländlichen Gebieten verbessert, Projekte für besonders benachteiligte Schüler und gegen den frühzeitigen Schulabbruch angestoßen, die Inklusion von Gitano-Schülern forciert (Bekämpfung des Absentismus, Anhebung des Schulleistungsniveaus, Einstellung von spezialisierten Lehrkräften), sowie die Bedürfnisse itineranter Gruppen (Schausteller, Wanderarbeiter) stärker berücksichtigt (Ministerio de Educación y Ciencia, 1990). Die kompensatorische Bildung, die in dieser Form bis etwa zum Jahr 2000 die schulpolitische Richtung wies, wurde von der sozialistischen Regierung als Politik der „positiven Diskriminierung“ verstanden, die das „Konzept der Chancengleichheit in der Bildung neu definiert" habe (Ministerio de Educación y Ciencia, 1993, zitiert nach Salinas, 2009, 177).

Mit der Jahrtausendwende stand auch der kompensatorische Ansatz in der Kritik. Die Asociación de Enseñantes con Gitanos etwa kritisierte in einem Dokument, dass sich durch die kompensationspädagogischen Maßnahmen viele Fehler der Brückenschulen wiederholten. So trage die kompensatorischen Bildungspolitik zur schulischen Segregation bei, erkläre individuelle Schüler als defizitär und habe weder ausreichend zum angestrebten Schulerfolg der Kinder und Jugendlichen beigetragen, noch etwas gegen die Marginalisierung und Diskriminierung der Gitanos in der Gesellschaft beigetragen (Salinas, 2009, 178). Kritik kam auch aus der Wissenschaft. Autoren wie Mariano Fernández Enguite vertraten die Ansicht, die Bildungsmisere der Gitanos sei in erster Linie eine Konsequenz ihrer Armut und kein kulturelles Problem, dass sich mit Kompensationspädagogik lösen ließe; andere forderten eine stärkere curriculare Öffnung und die Verankerung interkultureller Ansätze in Pädagogik und Schulpraxis (Salinas, 2009, 178ff.).

Gegenwärtig folgt die Beschulung von Kindern und Jugendlichen aus Gitano-Familien einem inklusiven Modell, welches parallel zum zunehmend heterogenen Charakter der spanischen Schulen den Stellenwert interkultureller und inklusiver Ansätze in Bildungspolitik und Bildungspraxis widerspiegelt. Alle Kinder und Jugendlichen aus Gitano-Familien besuchen die allgemeinen Schulen oder (zu einem sehr geringen Prozentsatz) Privatschulen. Für Schüler mit Lernschwierigkeiten gleich welcher Herkunft - stehen Förderangebote zur Verfügung. Diese Förderung wird von Lehrkräften durchgeführt, die unter der Leitung von Schulpsychologen individualisierte Lernpläne für die einzelnen Schüler erstellen, die dazu dienen sollen, besser auf die individuellen Lernunterschiede der Schüler einzugehen, ohne sie in separaten Klassen oder Schulen zu unterrichten. Wenn Lernrückstände von mehr als zwei Schuljahren diagnostiziert werden, wird geprüft, ob bei den 
betreffenden Kindern „besondere pädagogische Bedürfnisse“ (necesidades educativas especiales) vorliegen. Den Schulen und Lehrkräften stehen verschiedene offizielle Angebote zur Verfügung, um ihre pädagogische Arbeit mit Gitanos zu unterstützen. So verantwortet der Arbeitsbereich für inklusive Bildung (Educación inclusiva) im Bildungsministerium mehrere Programme, mit denen ein Beitrag zur Inklusion und zur Chancengleichheit bestimmter Schülergruppen geleistet werden soll. Darunter befindet sich das Bildungsprogramm für das Volk der Gitanos (Programa de Educación del Pueblo Gitano), das in Zusammenarbeit der Arbeitsstelle für interkulturelle Bildung des Bildungsministeriums und dem Ministerium für Arbeit und Soziales entwickelt wurde. Zu den verschiedenen Aufgaben des Programms gehört die Herausgabe von didaktischem Material zur Kultur der Gitanos, die interkulturelle Bildung und Mediation sowie die Aus- und Fortbildung von Lehrkräften und anderen Personen, die in pädagogischen Handlungsfeldern tätig sind und mit Gitanos zusammenarbeiten.

Zusätzlich ist die FSG landesweit mit mehreren einflussreichen Programmen und Kampagnen zur Verbesserung der Bildungsbeteiligung von Gitanos aktiv. Man arbeitet u.a. an (a) der Förderung und Unterstützung des Besuchs frühpädagogischer Institutionen, (b) der „Normalisierung“ der Bildungsbeteiligung von Gitano-Jugendlichen im schulpflichtigen Alter (v.a. im Rahmen des sogenannten Promociona-Programms), (c) einer engagierteren Einbindung von Gitano-Familien in den Bildungsprozess ihrer Kinder, (d) der Dokumentation und Analyse der Schulsituation von Gitanos durch empirische Forschungen und Publikationen; (e) Angeboten der Erwachsenenbildung für Gitanos; (f) der Fortbildung und Unterstützung für Pädagogen und Sozialarbeiter; (g) der Entwicklung unterschiedlicher Bildungs- und Anti-Diskriminierungskampagnen, sowie (h) der Entwicklung und Partizipation an internationalen Roma-Projekten.

\section{Bildungsdisparitäten im Wandel: Ausgewählte empirische Entwicklungen}

Die Bildungsdisparitäten zwischen der Gitano-Minderheit und der spanischen Mehrheitsgesellschaft sind empirisch gut belegt und in der Fachdiskussion prinzipiell nicht umstritten (vgl. z.B. Abajo Alcalde \& Carrasco, 2004; Alfageme Chao \& Martínez Sancho, 2004; Asociación Secretariado General Gitano, 2001; Beuchling, 2010; Gamella, 2012; Equipo EINA, 2003; IFIIE \& Instituto de la Mujer, 2010; Instituto de la Mujer, CIDE \& Fundación Secretariado Gitano, 2006). Zugleich kann gezeigt werden, dass in den letzten Jahren ein Wandel innerhalb der Gitano-Gemeinschaften eingetreten ist und sich ihre Bildungssituation graduell verbessert.

\section{Analphabetismus}

Die Befähigung, Lesen und Schreiben zu können, ist in modernen Gesellschaften Voraussetzung für die Teilhabe an zahlreichen Aktivitäten des beruflichen, sozialen und kulturellen Lebens. Fehlende oder mangelnde literale Kompetenz hat gravierende Auswirkungen auf die Beschäftigungsmöglichkeiten, die Fähigkeit, den Anforderungen des täglichen Lebens selbsttätig nachzukommen und den individuellen Bildungs- und Kenntnishorizont. Analphabetismus begünstigt damit Abhängigkeitsverhältnisse zu anderen Menschen und gesellschaftlichen Institutionen.

Verglichen mit anderen europäischen Ländern hatte Spanien bis Mitte des vorigen Jahrhunderts eine relativ hohe Analphabetenrate. Noch 1950 betrug der Anteil der Analphabeten in Gesamtspanien 17,3 Prozent und lag in ländlichen und stärker agrarisch geprägten Regionen noch höher (Höffer-Mehlmer, 2009, 52). Seitdem ist der Analphabetismus stark zurückgegangen. Im Bevölkerungszensus des Nationalen Instituts für Statistik von 2008 wird die Zahl der illiteraten Personen über 15 Jahren mit 872436 angegeben, was einem Anteil von 2,2 Prozent dieser Altersgruppe 
entsprach. Besonders betroffen waren Frauen, Menschen über 60 Jahre, Bewohner Südspaniens sowie Angehörige der Gitano-Minderheit (letztere werden in Erhebungen des NIS nicht erfasst).

Der Analphabetismus junger Gitanos ist gegenüber der Generation der Eltern und Großeltern deutlich zurückgegangen, liegt im Vergleich zur spanischen Mehrheitsbevölkerung immer noch hoch. Ein bedeutender Teil der Gitanos sind Analphabeten, Frauen häufiger als Männer, ältere Menschen häufiger als jüngere. Offizielle Hochrechnungen gehen davon aus, dass landesweit bis zu 16,4 Prozent der jungen Gitanas und 9,6 Prozent der jungen Gitanos lese- und schreibunkundig sind (Ministerio de Sanidad, Política Social y Igualdad, 2010).

In Andalusien, der Region mit der höchsten Analphabetenrate in Spanien, fand die periodisch durchgeführte Familienbefragung des Nationalen Instituts für Statistik (Encuesta de Población Activa) rund 278000 Personen im Alter über 15 Jahre, die weder lesen noch schreiben konnten. Dies entspricht einer Rate von über 4 Prozent. Der Anteil der Lese- und Schreibunkundigen war bei den Frauen mehr als doppelt so hoch wie bei den Männern; bei den Älteren über 65 Jahren betrug er 17,3 Prozent. Am stärksten ausgeprägt war der Analphabetismus bei Frauen über 65 Jahre mit 22,4 Prozent (Encuesta de Población Activa, 2007, zitiert nach Gamella, 2012, 111). Diese altersund geschlechtsspezifischen Unterschiede in der andalusischen Bevölkerung spiegeln sich auch in der Alphabetisierung der Gitanos wider. Gamellas eigene Feldstudien in Andalusien zeigen, dass der Analphabetismus unter den Gitanos in den stark von Gitanos bewohnten Ortschaften um Granada deutlich höher ist als in der Gesamtregion, wobei der Anteil lese- und schreibunkundiger Gitanos in den untersuchten andalusischen Dörfern zwischen 8 und 35 Prozent beträgt (ebd.).

\section{Bildungsbeteiligung}

Die aktuellsten Hochrechnungen zur Bildungsbeteiligung bzw. Beschulungsrate von Gitanos liegen mittlerweile zehn Jahre zurück. Demnach gab es im Schuljahr 2001/2002 in Spanien 16789 Primar- und Sekundarschulen, in nahezu der Hälfte dieser Schulen befanden sich Kinder und Jugendliche aus Gitano-Familien. Insgesamt gingen 119339 Gitano-Schüler in die Vorschulen, Primarschulen und Sekundarschulen, was etwa 2,2 Prozent der Gesamtschülerschaft Spaniens entsprach. Am stärksten waren Gitano-Kinder mit 69078 Lernenden (2,8 Prozent) im Primarbereich vertreten. Im Sekundarbereich fiel die Zahl auf 22442 Lernende (1,2 Prozent). Der überwiegende Teil der Gitano-Schüler besuchte staatliche oder vom Staat maßgeblich unterstützte Schulen. Lediglich 3,8 Prozent waren in Privatschulen eingeschrieben. Ihre Verteilung variierte in den Schulen stark. In 32 Prozent der Schulen waren weniger als zehn Gitano-Kinder eingeschrieben, in 15 Prozent der Schulen gab es eine größere Anzahl, während sie in 1,7 Prozent der Schulen über die Hälfte der Schülerschaft ausmachten (Giménez Adelantado, 2003; zu ähnlichen, geringfügig höheren Gesamtzahlen kommt die ebenfalls empirisch untermauerte Hochrechnung von Alfageme Chao und Martínez Sancho (2004)).

\section{Einschulungsalter, vorschulischer Bereich und Primarbereich}

Das Alter, in dem Gitano-Kinder das erste Mal eine Einrichtung des spanischen Bildungssystems (Kindergarten, Vorschule, Primarschule) besuchen, ist deutlich gesunken. 2009 waren 93,2 Prozent der Gitano-Kinder zum Zeitpunkt ihrer Einschulung sechs Jahre oder jünger. Damit kam ein Großteil der Eltern mittlerweile der Schulpflicht nach, die in Spanien im Alter von sechs Jahren beginnt. Fast die Hälfte der beschulten Kinder wurde bereits im Alter von drei Jahren eingeschult, was zeigt, dass Gitano-Eltern frühpädagogische und vorschulische Angebote zunehmend nutzen. Angesichts des Stellenwertes der frühkindlichen Förderung zum Ausgleich von Benachteiligungseffekten wird diese Tendenz positiv verzeichnet. Zudem ging die Anmeldung in 89 Prozent der Fälle initiativ von 
der Familie aus. Von 6,8 Prozent der eingeschulten Kindern waren die Eltern der Schulpflicht verzögert nachgekommen: Diese Kinder wurden erst (zum Teil auf Druck der Sozialen Dienste) mit sieben Jahren oder später angemeldet. Damit zeichnet sich gegenüber den 1990er Jahren eine Verbesserung ab: 1994 lag der Anteil der fristgemäß eingeschulten Gitano-Kinder noch bei 79 Prozent, 21 Prozent waren sieben Jahre oder älter (Fundación Secretariado Gitano, 2010, 27ff.).

Dem schulischen Absentismus (überdurchschnittlich häufiges, z.T. unentschuldigtes Fehlen) kommt im spanischen Kontext ein außerordentlich hoher Stellenwert zu, was verschiedene, auch historische Gründe hat. Da die regelmäßige Teilnahme am Unterricht große Auswirkungen auf die Motivation, die Beziehungen zu Mitschülern und Lehrkräften und v.a. auf die Schulleistungen hat, ist es naheliegend, den Absentismus empirisch zu untersuchen. Generell kann festgehalten werden, dass für Kinder aus Gitano-Familien verschiedentlich sehr hohe Fehlzeiten attestiert wurden. Obgleich das Phänomen im Sekundarbereich gravierender wird, lässt es sich bereits in den Primarschulen (Klassen 1-6) aufzeigen. Im Schuljahr 1994 fehlten noch 57 Prozent der GitanoPrimarschüler über längere Zeiträume. Im Schuljahr 2009 war der Anteil der Gitano-Kinder, die drei Monate oder länger fehlten, auf 22,5 Prozent zurückgegangen. Die Mädchen wiesen etwas niedrigere Absentismusraten auf als die Jungen. Eine detaillierte lokale Studie zum Absentismus von Gitano-Kindern in einer Primarschule in Salamanca konnte zudem deutliche Variationen zwischen den Schuljahren identifizieren. Aus ungeklärten Gründen erreichte der Absentismus bereits in der zweiten Klasse seinen höchsten Wert, wo 49,1 Prozent der Gitano-Kinder nicht einmal der Hälfte aller Schulstunden in dem Schuljahr beiwohnten. Ebenso ungeklärt ist, warum in der Untersuchung die Monate März und November die Monate mit den meisten Fehlzeiten waren (Manjón Barrera, 2011).

\section{Sekundarstufe - ESO}

Übergänge von einem Schultyp in den nächsthöheren bilden Schwellensituationen, in denen sich statistisch oftmals Selektionssituationen manifestieren. In der Bildungssituation der Gitanos in Spanien bildet der Übergang von der Primarschule in die schulpflichtige Sekundarschule (ESO) nach der Klasse 6 eine solche Schwellensituation. Gitano-Mädchen vollziehen den Schritt von der Primar- zur Sekundarschule trotz besserer Leistungen in der Grundschule seltener als GitanoJungen: Im ersten Schuljahr der Sekundarschule sind Gitano-Mädchen mit 39,3 Prozent klar unterrepräsentiert. Im Verlauf der Schuljahre verändert sich das Zahlenverhältnis jedoch. Obgleich der Anteil der Gitanos unter den Sekundarschülern generell sinkt, findet der Abbruch der Schullaufbahn bei den männlichen Jugendlichen nun häufiger statt, sodass die Mädchen im zweiten Schuljahr der ESO bereits 43,7 Prozent der Gitano-Schülerschaft in der ESO repräsentieren und im dritten Schuljahr auf genau 50 Prozent aufgeschlossen haben. Im letzten Jahr der schulpflichtigen Schulzeit haben die Gitano-Mädchen schließlich den Prozentsatz vom ersten Jahr umgekehrt und besuchen mit 63,4 Prozent häufiger die ESO als ihre männlichen Pendants. Obgleich anteilig weniger Mädchen mit der ESO beginnen, liegt die Wahrscheinlichkeit, dass sie die Pflichtschulzeit erfolgreich beenden, höher als bei den Jungen. Relativiert wird dieser Umstand jedoch durch die absoluten Zahlen: In dem landesweiten Sample der Erhebung der Fundación Secretariado Gitano waren es nur noch 26 Mädchen aus einer Stichprobe von 407 Gitano-Jugendlichen (vgl. Fundación Secretariado Gitano, 2006a, 32, sowie Beuchling, 2010, 92). Von den jugendlichen Gitanos, die sich im ersten Schuljahr der ESO befanden, beendeten 80 Prozent nicht das letzte Jahr der schulpflichtigen Sekundarstufe.

Die Begründungen für die hohen Fehlzeiten sind nicht nur vielfältig, sondern schwierig zu identifizieren oder zu isolieren. So müssen die von den Schülern, ihren Eltern oder den Lehrkräften angeführten Begründungen nicht mit den tatsächlichen Gründen übereinstimmen oder mehrere 
Gründe konfundieren. Für eine relativ kleine Stichprobe in Andalusien, bei der geschlechtsspezifische Aufschlüsselungen fehlen und die keine Verallgemeinerung erlauben, wurden 2001 folgende Gründe genannt:

Tabelle 2: Gründe von Gitano-Schülern, dem Unterricht fernzubleiben, 2001 in Prozent (N=261)

\begin{tabular}{|c|c|}
\hline Die Familie ist zeitweise abwesend/verreist & $30,6 \%$ \\
\hline Desinteresse/Desorganisation der Familie oder der erwachsenen Erziehungsberechtigten & $25,0 \%$ \\
\hline Die Kinder arbeiten innerhalb oder außerhalb des Elternhauses & $11,1 \%$ \\
\hline Erkrankung des Schülers & $11,1 \%$ \\
\hline Die Familie sieht wenig Nutzen in der Schule, Lesen und Schreiben genügt & $7,4 \%$ \\
\hline Die Gründe sind nicht bekannt & $14,8 \%$ \\
\hline
\end{tabular}

\section{Schulleistungen}

Als Konsequenz aus den häufigen Fehltagen weisen Gitano-Schüler durchschnittlich schwächere Leistungen auf. Auch wenn in den international vergleichenden Schulleistungsstudien, an denen Spanien teilnahm, die ethnische Herkunft der getesteten Schüler nicht erfragt wurde, so gibt es andere Indikatoren, die ethnische Leistungsunterschiede anzeigen. Mitte der 1990er Jahre betrug der Anteil der Gitanos an den kompensatorisch geförderten Kindern 36 Prozent (bei einem Bevölkerungsanteil von maximal zwei Prozent), wobei allerdings große regionale Schwankungen verzeichnet wurden: In den Schulen der nordafrikanischen Enklave Melilla etwa waren es zu 97 Prozent Kinder nordafrikanischer Herkunft, die in den Kompensationsprogrammen waren, während es auf den Balearen wiederum zu 64 Prozent Gitanos waren (Grañeras Pastrana et al., 1999, 117). Gegenwärtig weisen 68,4 Prozent der Gitano-Schüler gegenüber 25,8 Prozent der Nicht-GitanoSchüler einen Lernrückstand von über zwei Jahren auf, so dass man bei ihnen „besondere pädagogische Bedürfnisse" diagnostiziert. Zudem bestehen nur 31,9 Prozent der Gitano-Schüler alle Fächer am Ende des Schuljahres, während dies bei den übrigen Schülern 58,1 Prozent sind (IFIIE \& Instituto de la Mujer, 2010).

\section{Vorzeitiger Schulabbruch}

Ein weiterer gravierender Nachteil liegt in der sehr hohen Häufigkeit des Schulabbruchs. Von den Gitano-Jugendlichen, die mit der ersten Klasse der Sekundarschule (7. Klasse) beginnen, beenden ca. 80 Prozent nicht mehr das letzte Jahr der Pflichtschulzeit (10. Klasse). Gitano-Mädchen verlassen die Schule vor allem beim Übergang von der Primar- zur Sekundarstufe, so dass im ersten Schuljahr der Sekundarschule bereits deutlich weniger Mädchen als Jungen anzutreffen sind. Dies ist umso problematischer, als dass Gitano-Mädchen, die die Schule besuchen, gemessen an ihrem Lernverhalten, ihrer Zufriedenheit und ihren Leistungen größere Erfolgsaussichten auf einen Schulabschluss aufweisen als die Jungen. Die Ursachen des Schulabbruchs unterscheiden sich bei Mädchen und Jungen. Bei den Mädchen werden v. a. der Druck durch die Familien und andere Angehörige der Gitano-Bezugsgruppen genannt, das negative Bild, das sich Familien von der Schule konstruieren, die Verpflichtung zu Hausarbeit und Kinderbetreuung sowie die Wahrnehmung der Rolle als Gitano-Frau. Bei den Jungen handele es sich eher um einen Mangel an Motivation und um das Interesse, einer bezahlten Arbeit nachzugehen. Zudem fehlen den Jugendlichen Bezugspersonen, die mit ihrer Bildung und ihrer Erwerbsbeschäftigung in mittel oder hoch qualifizierten Bereichen als Vorbilder bzw. soziale Modelle fungieren. Ebenso beeinflussen die wirtschaftlichen Ressourcen der Familien die Bildungslaufbahn, in der Regel wird jedoch die kulturelle Praxis gegenüber der öko- 
nomischen Praxis unterschätzt (vgl. Abajo Alcalde \& Carrasco, 2004; Beuchling, 2010; IFIIE \& Instituto de la Mujer, 2010).

\section{Hochschulstudium}

Hinsichtlich des Universitätsbesuchs junger Gitanos existieren keine verlässlichen Zahlen. In offiziellen Veröffentlichungen wird geschätzt, dass landesweit ungefähr 200 Gitanos über einen Universitätsabschluss verfügen. Für das Universitätssemester 2004/2005 ging man davon aus, dass von den 1462771 Studierenden an Spaniens Hochschulen weniger als 1000 Studierende Gitanos waren. Damit wären sie deutlich unterrepräsentiert. Ihrer demographischen Struktur zufolge müssten bei gleicher Studiumsquote über 28000 Gitanos studieren (Ministerio de Sanidad, Política Social y Igualdad, 2010). Einige Gitano-Organisationen widersprechen der genannten Zahl von 200 Akademikern vehement und vermuten, dass „Tausende Gitano-Studenten“ ein Hochschulstudium abgeschlossen hätten (Salinas, 2007, 44). Belege oder Begründungen für die jeweils geschätzte Anzahl fehlen. Die landesweite Befragung des Centro de Investigaciones Sociológicas von 1610 GitanoHaushalten kommt auf einen Akademikeranteil von 0,3 Prozent, was deutlich über den angeführten 200 Hochschulabsolventen läge (CIS, 2006/2007; vgl. Gamella, 2012, 97). Da die Stichprobe der CIS-Befragung zudem Gitano-Haushalte befragte, in deren Nachbarschaft sich mindestens zehn weitere Gitano-Haushalte befanden, ist es möglich, dass sie den Akademikeranteil eher unterschätzt.

\section{Bildungsdisparitäten und Ethnizität im Wandel: Resümee und weiterführende Anmerkungen}

Die Bildungssituation der Gitanos in Spanien hat sich in den vergangenen ca. drei Dekaden merklich verbessert. Dies wird an steigenden Beschulungs-, Alphabetisierungs- und Abschlussraten sowie einem nachlassenden Schulabsentismus sichtbar. Als Hintergründe dieser Verbesserung lassen sich eine generelle Transformation der spanischen Gesellschaft anführen (Demokratisierung, EUIntegration, Internationalisierung, Technologisierung und Stärkung von humankapitalintensiven Industrie- und Wirtschaftssektoren), die damit einhergehende Öffnung, Ausweitung und Modernisierung des spanischen Bildungssystems sowie eine Vielzahl staatlicher wie nichtstaatlicher Integrationsinitiativen, die zu einem graduellen Wandel der gitanischen Kultur beigetragen haben. Die Verbesserungen im Bildungsbereich sind eingebettet in eine generelle Verbesserung des Lebensstandards der Gitano-Bevölkerung. Relativiert wird der steigende Bildungsstand der GitanoBevölkerung durch den Umstand, dass sich im gleichen Zeitraum ebenso der allgemeine Bildungsstandard in Spanien in Folge der wachsenden Bedeutung humankapitalintensiver Wirtschaftsbereiche angehoben hat. Zu prüfen wäre, ob die Anhebung des Bildungsstandes in der Summe auch zu einer Verringerung der Bildungsdisparitäten zwischen Minderheit und Mehrheit geführt hat.

Dennoch sind nach wie vor deutliche Unterschiede zur Bildungssituation der Mehrheitsbevölkerung auszumachen, besonders prägnant bei den höheren Fehlzeiten von Kindern und Jugendlichen aus Gitano-Familien und dem geringeren Schulerfolg. Die Hintergründe dazu sind komplex, nicht zuletzt bedingt durch die Heterogenität der Gitano-Bevölkerung. Um diese Heterogenität zumindest im Ansatz nachzuvollziehen, ist es wichtig, nicht lediglich von einem ethnischen Kollektiv zu sprechen, sondern sich den Stellenwert familial-regionaler Netzwerke mit eigenen wirtschaftlichen Nischen vor Augen zu führen. In dem Bildungsgeschehen kommen dann die sozioökonomische Lebenslagen der Familien, lokale/regionale Besonderheiten des Wohnumfeldes, Qualität der Beziehungen zur Nachbarschaft und zu den Schulen, ethnokulturelle Praxis, Präferenzen und Akkulturationsprozesse, individuelle und familiale Verhaltensweisen, die dem Schulbesuch dienlich sind 
(strukturierte Tagesabläufe, Lern- und Arbeitsverhalten, soziale Kompetenzen...) oder eine bewusste Distanzierung und Ablehnung der Institutionen der „Payos“ zur Wahrung ethnischer Distinktivität und der Selbstbehauptung als Abstammungsgruppe zum Tragen.

So prekär die Lebenssituationen von vielen Gitano-Familien sind, so prekär ist auch die Bildungssituation. Von einer weitgehenden Konsolidierung oder Stabilisierung kann (zumindest für einen großen Teil der Heranwachsenden) bislang noch nicht gesprochen werden. Zu groß sind die Unterschiede, zu hoch die Fehlzeiten, zu gering die Anzahl der Gitanos, die die Schule mit einem Abschluss verlassen, zu notwendig ist noch das tägliche Engagement von Sozialarbeitern und NGOs. Am ehesten sind Konsolidierungsprozesse im Bereich der Alphabetisierung auszumachen, mithin in einem Kompetenzbereich, dessen Nutzen unmittelbar einsichtig ist (z.B. im Kontext von Handelsund Verkaufsaktivitäten, Führerscheinprüfungen, Behördenkontakten u. v. m.), die aber nicht an bestimmte Abschlüsse, Zeugnisse oder an die Vermittlung durch staatliche Schulen gebunden sind.

Die gegenwärtig größte Herausforderung für den Integrations- bzw. Inklusionsprozess der Gitano-Minderheit bildet die spanische Wirtschaftskrise seit 2007. Diese hat zum zu einer folgenschweren Arbeitslosigkeit von jungen Menschen geführt. Zwar lag die Erwerbslosigkeit von Heranwachsenden in Spanien schon früher relativ hoch, gegenwärtig findet aber über die Hälfte der Spanier unter 25 Jahren keinen Job - im August 2013 waren es 56 Prozent (Eurostat, 2013). Angesichts der Perspektivlosigkeit wandern viele junge Spanier ins Ausland aus, nehmen unbezahlte, aber umkämpfte Praktikumsstellen an oder gehen zurück ins Bildungssystem, um Abschlüsse nachzuholen. Zum anderen wurden durch die Wirtschaftskrise starke Einschnitte bei den öffentlichen Ausgaben notwendig, die auch den Bildungs- und Sozialsektor betreffen. Der Bildungssektor etwa ist von der Wirtschaftskrise in mehrerlei Hinsicht betroffen: Zur Reduzierung der Staatsverschuldung wurden u. a. die Lehrergehälter gekürzt und zahlreiche Investitionen in die Bildungsinfrastruktur eingefroren.

Einige Einblicke, wie die Gitano-Minderheit von der Krise betroffen ist, gibt ein im Frühsommer 2013 von der FSG auf Basis von Informationen aus 54 Regionalstellen der Stiftung erstellter Report (Fundación Secretariado Gitano, 2013). So ging mit dem Einbruch der Baubranche für männliche Gitanos ein wenige Jahre zuvor neu erschlossenes Erwerbsfeld verloren, geringqualifizierte GitanoFrauen seien hingegen etwas flexibler, da ihnen als Verkäuferinnen, Reinigungskräfte oder im ambulanten Handel etwas mehr Optionen offen stünden. Gegenwärtig sollen etwa 42 Prozent der erwerbsfähigen Gitano-Bevölkerung arbeitslos sein (ebd., 43). Die Einsparungen treffen auch Schüler aus Gitano-Familien (ebenso wie Schüler aus anderen Familien in sozioökonomisch belasteten Situationen) direkt: Die staatlichen Beihilfen für den Erwerb der Schulbücher und für das Essen in den Schulkantinen wurden in vielen der Autonomen Gemeinschaften gekürzt oder ganz gestrichen; mancherorts werden Schulbusse seltener eingesetzt, was erneut zu höheren Fehlzeiten in peripher gelegenen Wohngebieten geführt haben soll. Während viele junge Spanier durch die Wirtschaftskrise wieder in das Bildungs- und Ausbildungssystem zurückkehren, da ihnen der Zugang zum Arbeitsmarkt verschlossen bleibt, trage die Wirtschaftskrise dazu bei, dass wieder mehr jugendliche Gitanos versuchen, zu den finanziellen Ressourcen der Familie beizutragen und die Schule hintanstellen. Des Weiteren wurden Einsparungen bei speziellen Förder-/Kompensations- oder Unterstützungsprogrammen und bei der Erwachsenenbildung getätigt. Die FSG erachtet die spanische Wirtschaftskrise daher als gravierende Gefährdung der Fortschritte der Gitano-Integration, die in den Jahren um die Jahrtausendwende erreicht wurden:

\footnotetext{
„Bildung ist ein Schlüsselglied bei der sozialen Inklusion der Gitano-Gemeinschaft, da sie den Zugang zu zukünftigen Arbeitsplätzen und die ökonomische Unabhängigkeit von Personen bestimmt. Wenn dieses Bindeglied versagt, ist das gesamte Inklusionssystem betroffen; und die Sparmaßnahmen, die man ergriffen hat, vernachlässigen die am stärksten vulnerablen Gitano-Schüler. Langfristig können die Folgen dieser Bildungssituation gravierend sein. Die Kürzung der Schülerhilfen, der Essensbeihilfen, des
} 
Lernmaterials usw. betreffen allesamt sowohl die Teilnahme der Gitano-Schülerschaft im Klassenzimmer wie auch die Bildungsprozesse aller Gitano-Jungen und -Mädchen und ihre schulischen Lernerfolg; ein Teil der Schülerschaft hat nicht ausreichend Ressourcen, um die Kosten für Schulmaterial oder Schulessen aufzubringen. Diese Kürzungen produzieren eine Zunahme des schulischen Absentismus oder des Schulabbruchs, wie es in einigen Fällen bereits vor der Krise ein Problem darstellte. Größere Klassen und die Streichung von Förderklassen tragen dazu bei, dass Schüler mit Lernschwierigkeiten den Anschluss an den Leistungsstand ihres Jahrganges nicht erreichen können." (Fundación Secretariado Gitano, 2013, 43f., Übersetzung 0.B.)

\section{Literatur}

Abajo Alcalde, J.E. \& Carrasco, S. (2004): Experiencias y trayectorias de éxito escolar de gitanas y gitanos en España. Encrucijadas sobre educación, género y cambio cultural. Madrid: CIDE/Instituto de la Mujer.

Abajo Alcalde, J.E. (2004): Infancia gitana y paya: convivencia y conflictos en la escuela. Tabanque, 18, 97-116.

Abajo Alcalde, J.E. (1999): La situacion de los niños gitanos en España: un test a nuestro sistema social y escolar. In: Revista Interuniversitaria de Formación del Profesorado, 36, S. 57-69.

Alfageme Chao, A. \& Martínez Sancho, M. (2004): Estructura de edades, escolarización y tamaño de la población gitana asentada en España. In: REIS - Revista Española de Investigaciones Sociológicas, 106, S. 161-174.

Asensio Belenguer, A. (2011): Absentismo y abandon escolar. Una aproximación desde la perspective de género al studio de las alumnas de etnia gitana del IES María Moliner. In: Acciones $e$ Investigaciones Sociales, 29, S. 105-129.

Asociación Secretariado General Gitano (2001): Evaluación de la normalización educativa del alumnado Gitano en Educación Primaria. In: Gitanos - Revista Bimestral de la Asociación Secretariado General Gitano, 11. (Dossier Educación, 2).

Asociación Secretariado General Gitano ([1979] 1990): Estudio sociológico : los gitanos españoles 1978. Madrid: ASGG.

Beuchling, 0. (2012): Bildung als Adaptation? John U. Ogbu und der kulturökologische Ansatz der Sozialisations- und Minderheitenforschung. Berlin: wvb.

Beuchling, 0. (2011): Die Bildungssituation des pueblo gitano im heutigen Spanien. Vortrag auf der Tagung Bildungsperspektiven für Sinti und Roma des Verbandes Deutscher Sinti und Roma/Landesverband Baden-Württemberg e.V. am 13. und 14. Mai 2011. Evangelische Akademie Bad Boll.

Beuchling, 0. (2010): Zwischen Payos und Gitanos. Eine Studie zur ethnischen Bildungsungleichheit in Spanien. Münster: Waxmann.

Cardiel, B. (2007): Educación y comunidad gitana. In: Laparra, M. (Hrsg.) (2007) Situación social y tendencias de cambio en la Comunidad Gitana. Pamplona: Universidád Publica de Navarra, S. 4584.

Caselles Pérez, J. F. (2013): Vivienda y pueblo Gitano. In: Hernández Pedreño, M. (Hrsg.) Vivienda y exclusión residencial. Murcia: Universidad de Murcia, S. 269-287.

Caselles Pérez, J. F. (2008): Factores socials de la exclusion social del pueblo Gitano. In: Hernández Pedreño, M. (Hrsg.) Exclusión social y desigualdad. Murcia: Universidad de Murcia, S. 229-252..

CIS - Centro de Investigaciones Sociológicas (2006/2007): Estudio CIS no. 2664: Encuesta sociológica a hogares de la población gitana. Abgerufen unter http://www.cis.es/cis/opencm/ ES/1_encuestas/estudios/ver.jsp? estudio=7820 (5. November 2013).

Equipo EINA (2003): La escolarización de la infancia gitana en 167 poblaciones de Andalucía. Castelló de la Plana: Publicacions de la Universitat Jaume. 
Eurostat (2013): Arbeitslosenquote des Euroraum bei 12\%. Eurostat Pressemitteilungen, 140/2013. Abgerufen unter http://epp.eurostat.ec.europa.eu/cache/ITY_PUBLIC/3-01102013 -AP/DE/301102013-AP-DE.PDF.

Fernández Enguite, M. (1999): Alumnos gitanos en la escuela paya. Un estudio sobre las relaciones étnicas en el sistema educativo. Barcelona: Ariel.

Fernández Enguite, M., Martín Lluch, L. F., Suárez Galván, F., \& García Peréz, A. (1993): La escolarización del pueblo gitano. Unveröffentlichter Report. Madrid: CIDE.

Fernández Enguite, M., Mena Martínez, L. \& Riviere Gómez, J. (2010): Fracaso y abandono escolar en España. Barcelona: Fundación "la Caixa”.

Fresno, J. M. (2001/2002): Secretariado Gitano: treinta y siete años de historia. In: Gitanos - Revista Bimestral de la Fundación Secretariado General Gitano, 12 \& 13, S. 72-77.

Fundació Pere Tarrés (2005): Estudi sobre la població gitana de Catalunya. Informe final. Barcelona: Generalitat de Catalunya, Departament de Benestar I Família.

Fundación Secretariado Gitano (2013): El impacto de la crisis en la comunidad gitana. Madrid: Fundación Secretariado Gitano.

Fundación Secretariado Gitano (2012): Población gitana, empleo e inclusión social. Un studio comparado: Población gitana española y del este de Europa. Madrid: Fundación Secretariado Gitano.

Fundación Secretariado Gitano (2008/2009): Mapa sobre Vivienda y Comunidad Gitana en España, 2007. In: Gitanos, 47 \& 48, (Dossier Vivienda).

Fundación Secretariado Gitano. (2010): Evaluación de la Normalización educativa de las alumnas y alumnos gitanos en Educación Primaria. Madrid: Instituto de la Mujer \& IFIIE.

Fundación Secretariado Gitano (2006): Investigación: El acceso del alumnado gitano a la enseñanza secundaria. In: Gitanos - Revista Bimestral de la FSG, 34/35. (Dossier Educación, 4).

Gamella, J. F. (2012): Historias de éxito. Modelos para reducir el abandono escolar de la adolescencia gitana. Madrid: Ministerio de Educación.

Gamella, J. F. (2006): Oficios gitanos tradicionales en Andalucía (1837-1959). In: Gitanos - Revista Bimestral de la FSG, 32/33, S. 64-73.

Gamella, J. F. (1999): Los gitanos andaluces. Una minoría étnica en una encrucijida histórica. In: Demófilo - Revista de cultura tradicional de Andalucía, 30, S. 15-29.

Gamella, J. F. (1996): La población gitana en Andalucía. Un estudio exploratorio de sus condiciones de vida. Granada: Junta de Andalucía.

García Pastor, B. (2011). ¿Qué entienden las y los gitanos por educación? In: Cadernos de Educação, 39, S. 61-81.

García Pastor, B. (2009): "Ser gitano" fuera y dentro de la escuela: una etnografía sobre la educación de la infancia gitana en la ciudad de Valencia. Madrid: CSIC.

Garreta Bochaca, J. (2003): La integración sociocultural de las minorías étnicas (gitanos e inmigrantes). Barcelona: Anthropos.

Gay y Blasco, P. (2005): Love, Suffering, Grief Among Spanish Gitanos. In: Milton, K. \& Svašek, M. (Eds.) Mixed Emotions: Anthropological Studies of Feelings. Oxford: Berg, S. 164-177.

Gay y Blasco, P. (1999): Gypsies in Madrid: Sex, Gender and the Performance of Identity. Oxford: Berg. Giménez Adelantado, A. (2003): The Education of Gypsy Children in Europe. European Union supported Educational Research Final Report. Brussels: European Commission.

Gómez Alfaro, A. (2010): Escritos sobre gitanos. Barcelona: Ensenantes con Gitanos.

Grañeras Pastrana, M. et al. (1999): Las Desigualdades de la Educación en España, II. Madrid: Ministerio de Educación y Cultura / Centro de Investigación y Documentación Educativa.

Höffer-Mehlmer, M. (2009): Modernisierung und Sozialarbeit in Spanien. Bremen: Europäischer Hochschulverlag. 
Instituto de la Mujer, CIDE \& Fundación Secretariado Gitano (2006): Incorporación y trayectoria niñas gitanas en la E.S.O. Madrid: Instituto de la Mujer.

Lagunas Arias, D. (2002): Modern Gypsies: Gender and kinship among the Caló from Catalonia. In: Romani Studies 12 (1), S. 35-55.

Lalueza, J. L., Crespo, I., Pallí, C. \& Luque, M. J. (2001): Socialización y cambio cultural en una comunidad étnica minoritaria. El nicho evolutivo Gitano. In: Cultura y Educación, 13 (1), S. 115130.

Laparra, M. (Hrsg.) (2011): Diagnóstico social de la comunidad gitana en España. Un análisis contrastado de la Encuesta del CIS a Hogares de Población Gitana 2007. Madrid: Ministerio de Sanidad, Política Social e Igualdad.

Laparra, M. \& García, Á. (2011): Una comunidad gitana de tamaño y perfiles todavía imprecisos. In: Laparra, M. (Hrsg.) Diagnóstico social de la comunidad gitana en España. Un análisis contrastado de la Encuesta del CIS a Hogares de Población Gitana 2007. Madrid: Ministerio de Sanidad, Política Social e Igualdad, S. 27-34.

Laparra, M. (Hrsg.) (2007): Situación social y tendencias de cambio en la Comunidad Gitana. Pamplona: Universidád Publica de Navarra.

Laparra, M. \& del Pozo, J. M. (2007): Población y estructura demográfica de la comunidad gitana. In: Laparra, M. (Hrsg.) Situación social y tendencias de cambio en la Comunidad Gitana. Pamplona: Universidád Publica de Navarra, S. 11-23.

López de la Nieta, M. (2011): La inserción educativa de la comunidad gitana: ¿realidad o espejismo? In: Laparra, M. (Hrsg.) Diagnóstico social de la comunidad gitana en España. Un análisis contrastado de la Encuesta del CIS a Hogares de Población Gitana 2007. Madrid: Ministerio de Sanidad, Política Social e Igualdad, S. 73-114.

Manjón Barrera, S. (2011): Normalización educativa del alumnado gitano de educación primaria. Master Thesis, Facultad de Derecho, Universidad de Salamanca.

Martín Palomo, T. (2002): Mujeres gitanas y el sistema penal. In: La Ventana, 15, S. 149-174.

Martínez Sancho, M., Giménez Adelantado, A., \& Alfageme Chao, A. (2001): La situación escolar de la infancia gitana y el absentismo. Vortrag auf dem VII. Congreso Español de Sociología, 20.-22. September 2001, Universität Salamanca.

Matras, Y. (2003): Die Sprache der Roma: Ein historischer Umriss. In: Matras, Y., Winterberg, H. \& Zimmermann, M. (Hrsg.) Sinti, Roma, Gypsies: Sprache - Geschichte - Gegenwart. Berlin: Metropol, S. 231-261.

Mena Cabezas, I. R. (2007): Los gitanos y la vente ambulante. Una economía étnica singular. Factoria de Ideas. Sevilla: Centro de Estudios Andaluces. Abgerufen unter http://www.centrodeestudiosandaluces.es/datos/paginas/factoria/ideas/informe_gitanos.pdf (2. November 2009).

Ministerio de Educación, Política Social y Deporte (2007): Programa de Desarrollo Gitano. Memoria de Evaluación 2006. Madrid: Ministerio de Educación, Política Social y Deporte.

Ministerio de Educación y Ciencia (1990): Proyectos de Compensación Educativa en Centros de E.G.B. Madrid: Ministerio de Educación y Ciencia.

Ministerio de Sanidad, Política Social y Igualdad (2010): Plan de acción de para el desarrollo de la población gitana, 2010-2012. Madrid: Ministerio de Sanidad, Política Social y Igualdad.

Ministerio de Sanidad, Servicios Sociales e Igualdad (2012): Estrategia Nacional para la Inclusión Social de la Población Gitana en España 2012-2020. Madrid: Ministerio de Sanidad, Servicios Sociales e Igualdad, Centro de Publicaciones.

Ministerio de Sanidad y Consumo \& Fundación Secretariado Gitano (2005): Salud y Comunidad Gitana. Análisis de propuestas para la actuación. Madrid: Ministerio de Sanidad y Consumo.

Pape, U. (2008): Europäische Union: Erstes Roma-Gipfeltreffen. In: Migration und Bevölkerung Newsletter, 8, Oktober 2008, S. 1-2. 
Petísco, L. (2012): Los gitanos españoles han cambiado más en tres décadas que en seis siglos. $E l$ País vom 6. Februar 2012.

Pym, R. (2007): The Gypsies of Early Modern Spain, 1425-1783. Houndmills: Palgrave Macmillan.

Ringold, D., Orenstein, M. A. \& Wilkens, E. (2005): Roma in an Expanding Europe: Breaking the Poverty Cycle. Washington: The World Bank.

Salinas, J. (2009): Un viaje a través de la historia de la escolarización de las gitanas y gitanos españoles. In: Anales de Historia Contemporánea, 25, S. 165-188.

Salinas, J. (2007): Reflections on Educational Policies for Spanish Gypsies. In: European Education, 39 (1), S. 32-49.

San Román, T. (1990): Gitanos de Madrid y Barcelona. Ensayos sobre Aculturación y Etnicidad. Barcelona: Publicacions d'Antropologia Cultural, Universitat Autònoma de Barcelona.

\section{Über den Autor}

Dr. Olaf Beuchling, M.A., Erziehungswissenschaftler und Dozent, ist Research Fellow am Zentrum für Buddhismuskunde (Universität Hamburg/Deutschland), Mitglied EMIGRA Forschungsnetzwerk (Autonome Universität Barcelona/Spanien) und Herausgeber International Dialogues on Education. Kontakt: info@beuchling.de 